www.nature.com/pj

\title{
Three-dimensional analyses of spherulite morphology in poly(oxyethylene) and its blends with amorphous poly(d,I-lactic acid) using X-ray computerized tomography
}

\author{
Nguyen-Dung Tien ${ }^{1,2}$, Yukihiro Nishikawa ${ }^{3,4}$, Masato Hashimoto ${ }^{3}$, Masatoshi Tosaka ${ }^{5}$, \\ Sono Sasaki ${ }^{1,4,6}$ and Shinichi Sakurai ${ }^{1,4,6}$
}

Here, we report on the three-dimensional structural analyses of huge spherulites of poly(oxyethylene) (PEG) using X-ray computerized tomography (CT) in blends of PEG and amorphous poly(d,-lactic acid). Large spherulite formation is characteristic of PEG, and its direct observation using X-ray $C T$ is reported here for the first time. Slit-shaped cracks that were straight or curved were clearly observed using X-ray CT. Furthermore, scanning electron microscopic observation revealed that the cracks were parallel to bundles of lamellar crystallites. From these observations, we conclude that a set of radial cracks observed by $\mathrm{X}$-ray $\mathrm{CT}$ is a signature of a huge spherulite. Several aspects that are representative of an axialite structure are presented, and they are in good agreement with the intuitively proposed structural model for axialite long ago, although the size scale is much larger in our study.

Polymer Journal (2015) 47, 37-44; doi:10.1038/pj.2014.83; published online 19 November 2014

\section{INTRODUCTION}

Spherulites are commonly found in various materials, including mineral aggregates, ${ }^{1-3}$ simple organic liquids ${ }^{4,5}$ and polymers. ${ }^{6,7} \mathrm{~A}$ typical spherulite consists of needle-like crystals that diverge from the center of the spherulite and is often found in inorganic materials and small organic molecules. ${ }^{8-10}$ In those cases, the needle-like crystals are aligned radially from the center of the spherulite to provide a perfectly symmetrical shape. As for polymer spherulites, it is known that they consist of plate-like crystals, which are referred to as lamellae with their basal surfaces aligned parallel to the radial direction of the spherulite. A single lamella is first generated in the melt, and subsequent spawning of new lamellae occurs. The lamellae grow two-dimensionally in the direction parallel to their basal surface while maintaining an almost constant thickness. Through splitting and branching, spherically symmetric aggregates are eventually formed. Therefore, characteristic features occur near to the spherulite center, which do not appear in low-molar mass systems. These features include a sheaflike aggregate, known as an axialite or hedrite. ${ }^{11-13}$ The axialite structure has been found near the center of polymer spherulites ${ }^{14,15}$ by polarized optical microscopic observation of polymer melts sandwiched between coverslips. However, this microscopy technique is only applicable in two-dimensional crystallization, and experimental confirmation of axialites in three-dimensions is required. Khoury and Passaglia ${ }^{14}$ previously demonstrated the existence of the axialite at the center of a polymer spherulite in three dimensions using surface-replica transmission electron microscopy (TEM) of the fractured surface of a bulk sample. They presented two typical TEM micrographs demonstrating several aspects of axialites viewed from different perspectives. Unfortunately, in principle, it is impossible to observe different aspects originating from a single axialite using the surface-replica TEM method. Therefore, such a method is insufficient to characterize three-dimensional (3D) axialite structures, and a nondestructive 3D structural analysis technique is still required.

Recently, high-contrast X-ray computerized tomography (CT) has been optimized for use with polymeric materials, ${ }^{16}$ and several observations of the polymer blends have been reported. ${ }^{17}$ Its spatial resolution is $\sim 3 \mu \mathrm{m}$ at present, which is not high enough for visualization of spherulites or axialites. Nevertheless, we have successfully observed axialites in a poly(oxyethylene) (PEG) spherulite because of their large size. The formation of huge spherulites is characteristic of PEG in both pure PEG samples and in blends of PEG with the racemic random copolymer poly(d,l-lactic acid) (PDLLA), ${ }^{18,19}$ and direct observation of a 3D spherulite using X-ray $\mathrm{CT}$ is reported here for the first time. We describe in detail how the

${ }^{1}$ Department of Biobased Materials Science, Kyoto Institute of Technology, Kyoto, Japan; ${ }^{2}$ Venture Laboratory, Kyoto Institute of Technology, Kyoto, Japan; ${ }^{3}$ Department of Macromolecular Science and Engineering, Kyoto Institute of Technology, Kyoto, Japan; ${ }^{4}$ Polymer Material Science Research Group, Kyoto Institute of Technology, Kyoto, Japan; ${ }^{5}$ Institute for Chemical Research, Kyoto University, Kyoto, Japan and ${ }^{6}$ Polymer Materials Science Research Group, Kyoto Institute of Technology, Kyoto, Japan

Correspondence: Professor S Sakurai or Dr Y Nishikawa, Department of Biobased Materials Science, Kyoto Institute of Technology, Matsugasaki, Sakyo-ku, Kyoto 606-8585, Japan. 
spherulites and axialites can be identified using X-ray CT with the assistance of scanning electron microscopy (SEM).

\section{EXPERIMENTAL PROCEDURE}

PEG was purchased from Wako Pure Chemical Industries, Ltd. (Kyoto, Japan) with a weight-average molecular weight $\left(M_{\mathrm{w}}\right)$ of $2 \times 10^{4}$. PDLLA was purchased from Sigma-Aldrich Co. with $M_{\mathrm{w}}$ in the range of $75000-120000$. All chemicals were used as received without further purification.

The 100\% PEG sample and its blends with PDLLA at 20 and $50 \mathrm{wt} \%$ (referred to as DL20 and DL50, respectively) were dissolved in dichloromethane (DCM), yielding a solution with $\sim 5 \mathrm{wt} \%$ polymer concentration. The as-cast samples were prepared by solution casting for $\sim 24 \mathrm{~h}$ at room temperature. The evaporation rate of DCM was suppressed by covering the casting Petri dish with aluminum foil with small holes. During casting, solution weight was measured using electronic balance (GR-202, A\&D, Tokyo, Japan), and several photographs were taken with a digital camera. The resulting as-cast samples were subjected to X-ray CT (FLEX-M345, Beamsense, Osaka, Japan). The X-ray generator tube was operated at $40 \mathrm{kV}$, and the transmission images were acquired using a charge-coupled device (CCD) with a CsI scintillator. To construct a $3 \mathrm{D}$ image, the sample was rotated over $180^{\circ}$ at $0.25^{\circ}$ intervals. At each rotation angle, four transmission images were taken with the exposure time of $1 \mathrm{~s}$, and they were averaged to provide a total exposure time of $4 \mathrm{~s}$. The obtained 720 transmission images were reconstructed into a 3D image using a standard filtered back-projection algorithm. ${ }^{20}$ SEM was also conducted to observe the fractured sample surface. The freeze-fractured surface was prepared by breaking the sample in liquid nitrogen and observed using a VE-7600 (Keyence, Osaka, Japan) operated at $10 \mathrm{kV}$. Before SEM observation, samples were coated with platinum using ion-sputter.

Polarized optical microscopy (POM) (Nikon Eclipse E600 POL) was used for observing the spherulite structure in melt-cooled samples. As-cast samples were sandwiched between two coverslips, placed on a hot stage (Linkam LK-600PM) at $70^{\circ} \mathrm{C}$ for $\sim 2 \mathrm{~min}$ and gradually cooled to room temperature. Spherulite growth in the 100\% PEG and in the blend samples was recorded with a CCD movie camera.

\section{RESULTS AND DISCUSSION}

Huge spherulites formed in solution-cast PEG samples

It can be observed in Figure 1 that a spherulite bearing a fibrous texture was formed in the $100 \%$ PEG sample cast from a DCM solution onto a glass plate. The diameter of the spherulite displayed in Figure 1 is $\sim 5 \mathrm{~mm}$, which is very large compared with typical polymer

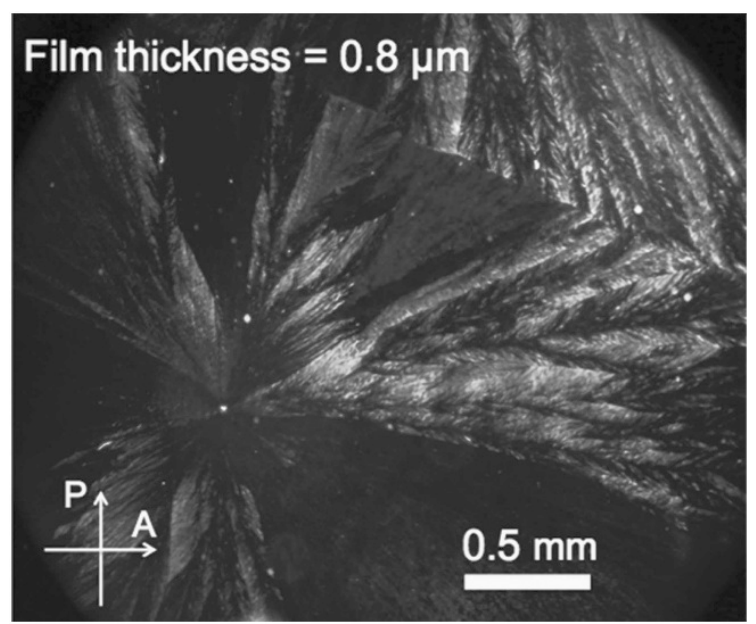

Figure 1 Polarized optical micrograph featuring a huge spherulite formed in a $100 \%$ PEG sample cast from a dichloromethane solution onto a glass plate. The spherulite diameter is estimated to be $\sim 5 \mathrm{~mm}$, and the aspect ratio is as large as 6250. A full color version of this figure is available at Polymer Journal online. spherulites (several tens of $\mu \mathrm{m}$ ). In addition, the aspect ratio (the diameter to thickness ratio) is also as large as 6250. Large spherulites were formed in sample cast from both DCM and aqueous solutions, for which a larger spherulite was formed with diameter up to $10 \mathrm{~mm}$ and aspect ratio of 455, as shown in Figure 2. Thus, solution-cast PEG characteristically forms large spherulites. It is also interesting to note that the same features can be observed using direct microscopic observation without crossed polarizers, as shown on the right panel of Figure 2. This effect may be due to the undulating surface of the fibrous-textured spherulites.

\section{Spherulite formation during solvent evaporation}

A plot of polymer solution concentration during solution casting for the $100 \%$ PEG sample versus elapsed time is shown in Figure 3. Corresponding photographs of the casting solutions and the as-cast samples are provided in Figure 4. In the plot in Figure 3, there are two kinks. Point (a) $(730 \mathrm{~min}$ elapsed) is coincident with the onset of crystallization, which was confirmed by the formation of white spots in the solution, as shown in Figure 4a. At kink point (b) (737 min

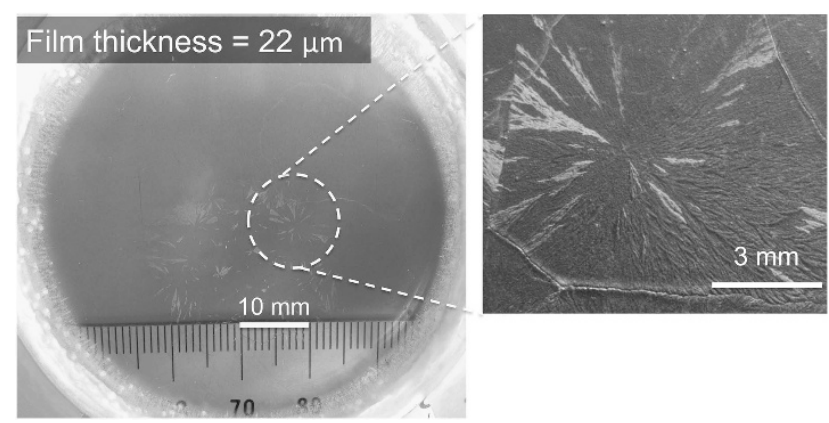

Figure 2 Bright-field image (by direct capturing with a CCD camera) featuring a huge spherulite formed in a 100\% PEG sample cast from an aqueous solution in a Petri dish. The spherulite diameter is $\sim 10 \mathrm{~mm}$, and the aspect ratio is 455 . A full color version of this figure is available at Polymer Journal online.

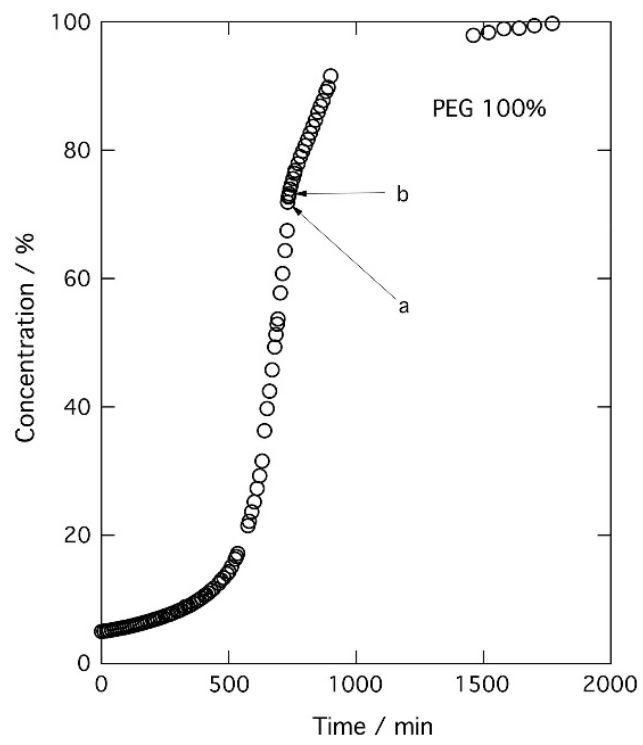

Figure 3 Plot of the casting solution (DCM solution) polymer concentration as a function of time for the 100\% PEG sample. 
a

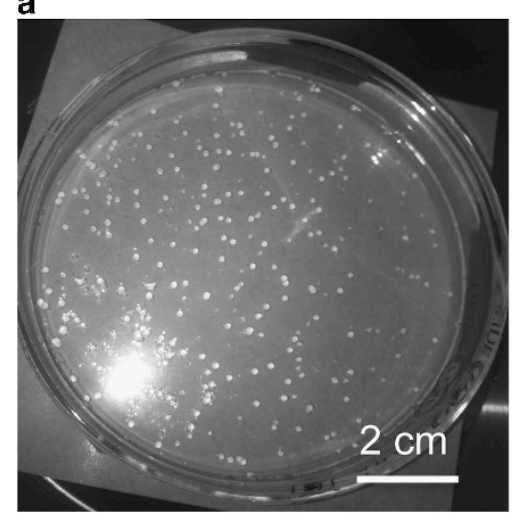

b

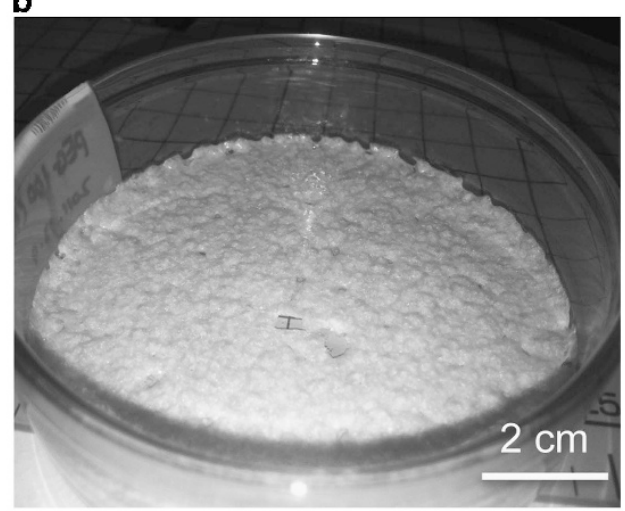

Figure 4 Photographs of the casting solution at $~ 730$ min corresponding to (a) the onset of crystallization and (b) after complete solvent evaporation (as-cast) for the $100 \%$ PEG sample. A full color version of this figure is available at Polymer Journal online.

elapsed), the white spots increased and space filled. Most likely, crystallization occurred near the solution surface due to the increase in surface polymer concentration and the cooling effect that was a result of the latent heat absorption due to solvent evaporation from the solution surface. Furthermore, the crystalline objects may continue to float near the surface, as the density of the PEG crystal $\left(1.235 \mathrm{~g} \mathrm{~cm}^{-3}\right)^{21}$ is smaller than that of the solvent, dichloromethane $\left(1.33 \mathrm{~g} \mathrm{~cm}^{-3}\right)$. After $900 \mathrm{~min}$, evaporation was further suppressed due to the reduction in the solvent partial pressure in the highly concentrated solution.

The as-cast sample surfaces were heavily bumped and cracked, as shown in Figure 4b. The surfaces of the as-cast samples with average thicknesses of $\sim 0.45 \mathrm{~mm}$ were found to be fully covered with bumps as large as $\sim 2-\mathrm{mm}$ width and $0.6-\mathrm{mm}$ height. The bumps are hypothesized to be a consequence of large spherulite formation, which motivated our conduction of X-ray CT analysis to further characterize the samples.

\section{X-ray CT analyses}

The as-cast samples were cut into small pieces, $\sim 1 \times 1 \times 0.3 \mathrm{~mm}^{3}$ and subjected to X-ray CT observation. Figures $5-7$ show the $3 \mathrm{D}$ and crosssectional images obtained using X-ray CT for the 100\% PEG as-cast sample, DL20 blend sample and DL50 blend sample, respectively.

As shown in Figure 5, many cracks were observed in regions A and B. Such characteristic cracks are hypothesized to be involved in large spherulites as the cracks appear to converge into a single point to form a spoke-like pattern in region A. This feature reminds us a spherulite. The cracks found in region B are different; however, it is possible to consider that the cracks converge into a single point that is located at the lower apex of the dotted lines outlining region $\mathrm{B}$. Thus, region $\mathrm{B}$ also appears to be part of a large spherulite. For the DL20 sample shown in Figure 6, the convergence of cracks is not clear. Furthermore, a large cavity and many voids and slit-like cracks were observed near the bottom surface of the sample (the surface in contact with the Petri dish). Thus, the features of the DL20 sample appear to be a bit different from those of the $100 \%$ PEG sample. However, the pattern of slit-like cracks can be identified in the region encircled with a white dotted line in Figure 6 that is similar to that observed in region B in Figure 5. Here, the converging point of the slit-like cracks may be located near the top of the bumped surface (as specified with a thick white arrow). If the converging point were the center of a spherulite, the cracks would be parallel to a bundle of lamellar crystallites. To examine this possibility, SEM observations were conducted, as shown

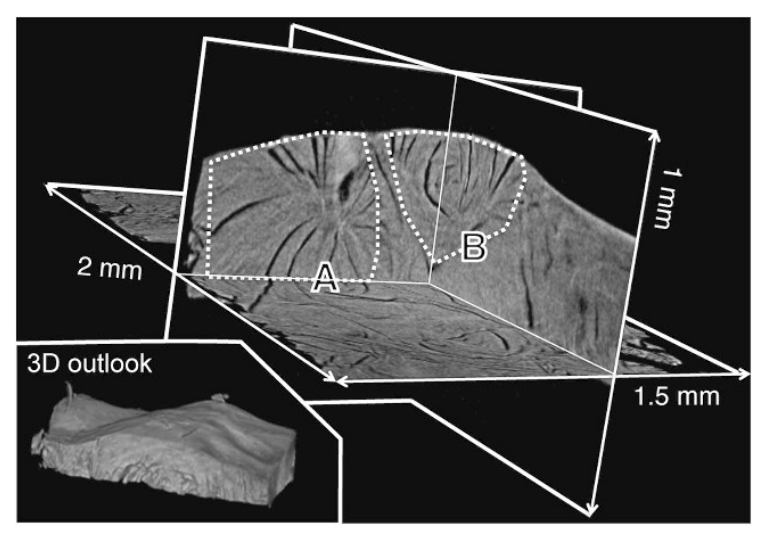

Figure $53 \mathrm{D}$ outlook (inset) and corresponding cross-sectional images obtained using X-ray CT for the 100\% PEG as-cast sample. The A and B regions highlight characteristic cracks that are involved in huge spherulites.

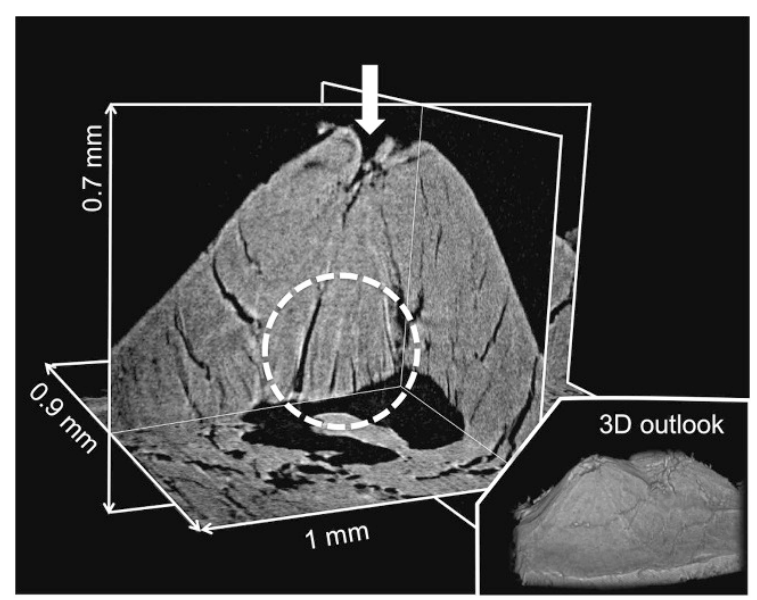

Figure $63 \mathrm{D}$ outlook (inset) and corresponding cross-sectional images obtained using X-ray CT for the DL20 as-cast sample.

in Figure 8, wherein the boundary between the spherulites can be clearly observed (Figure $8 \mathrm{~b}$ ). Therefore, we can conclude that the cracks are parallel to a bundle of lamellae in a spherulite. This conclusion in turn confirms that X-ray CT can detect the $3 \mathrm{D}$ 
structures of a cluster of cracks in large spherulites, as observed in regions A and B in Figure 5. Slit-shaped cracks were stochastically involved in the POM picture in Figure 9, showing a spherulite in the DL20 as-cast sample (cast on a glass plate by spin-coating at 2000 r.p. m. from a DCM solution; thickness of $\sim 1.4 \mu \mathrm{m}$ ). Here, cracks were located in the central region of the large spherulite. The POM image in Figure 9 was taken by inserting the retardation plate (optical retardation of $530 \mathrm{~nm}$ ) so that a yellow/orange color can be recognized in the upper-right and lower-left regions of the spherulite, and a blue/ purple color can be observed in the upper-left and lower-right regions. This coloring is typical of a negative spherulite. Figure 7 of the DL50 sample exhibits very different features from those of the 100\% PEG and DL20 samples. Many round voids can be observed. Although small cracks were observed near the top of the bump shown in the cross-sectional view, it is difficult to conclude that those cracks suggest the existence of spherulites, and it is likely that no large spherulites were formed due to the large amount of amorphous PDLLA blended into the DL50 sample. To determine why large spherulites were not formed in the DL50 sample, we conducted POM observation of DL50 on cooling from the melt (Figure 10).

Figure 10 shows time-sliced POM images of the development of crystallization in the DL50 sample melt, which was originally cast from a DCM solution in a Petri dish with thickness of $0.45 \mathrm{~mm}$. The as-cast sample was melted by heating at $70{ }^{\circ} \mathrm{C}$ (above $T_{\mathrm{m}}$ ) for $\sim 2 \mathrm{~min}$. The initial state of sample melt is shown in Figure 10a. Here, many bubbles of 10-20 $\mu \mathrm{m}$ size are observed that stayed at fixed positions and did not affect the observation of the crystallization process (Figures 10b-d).

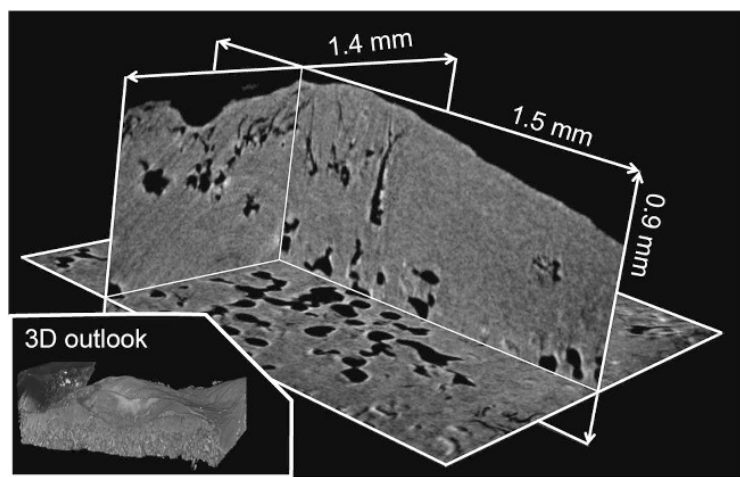

Figure $73 \mathrm{D}$ outlook (inset) and corresponding cross-sectional images obtained using X-ray CT for the DL50 as-cast sample.
Then, the sample was gradually cooled down to the room temperature. The elapsed time is labeled on each POM image in Figure 10. It can be observed that crystallization proceeded from the lower-right corner of the POM image with a partially curved frontline of crystalline growth region. It seems that spherulite formation began, but the growth front was split by small patches of $\sim 20-100 \mu \mathrm{m}$ diameters. Moreover, although the curvature of the frontline was large (that is, the hemispherical region in the vicinity of the growing front is small), close examination reveals that the hemi-spherical region exhibits a yellow/ orange color in the upper-right region and a blue/purple color in the upper-left region (Figure 10e), and other hemi-spherical regions show overlapping of the two colors. Thus, we might conclude that the negative spherulite was formed in the vicinity of the growth front. The formation of tiny spherulites implies that the capability of forming a spherulite of the PEG moiety in blend samples (even in the DL50 sample) is still active. Therefore, the reason for the absence of large spherulites in the DL50 sample is somewhat unclear. As stated above, patches such as those observed in Figure 10d may serve as obstacles to hinder the formation of large spherulites. Although crystallization proceeded over time, it can be observed that, in the round-shaped patches, the color remained unchanged over 306-s elapsed time (Figure 10d). This result implies that crystallization never took place in the round-shaped patches. Therefore, these regions (small patches) are considered to be phase-separated domains with an amorphous PDLLA-rich composition. Interestingly, these regions are visible even in Figure 10a, and they are clearly different from the cavity. The above explanation based on phase-separation is, at a first glance, not
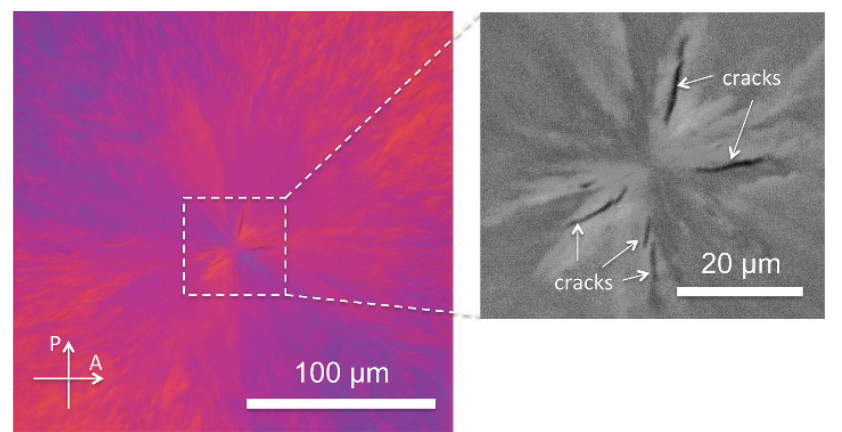

Figure 9 Polarized optical micrograph of cracks in the central region of spherulite formed in the DL20 sample cast from a dichloromethane solution onto a glass plate. A retardation plate with optical retardation of $530 \mathrm{~nm}$ was inserted. The magnified image on the right side was adjusted to gray scale to better observe cracks.
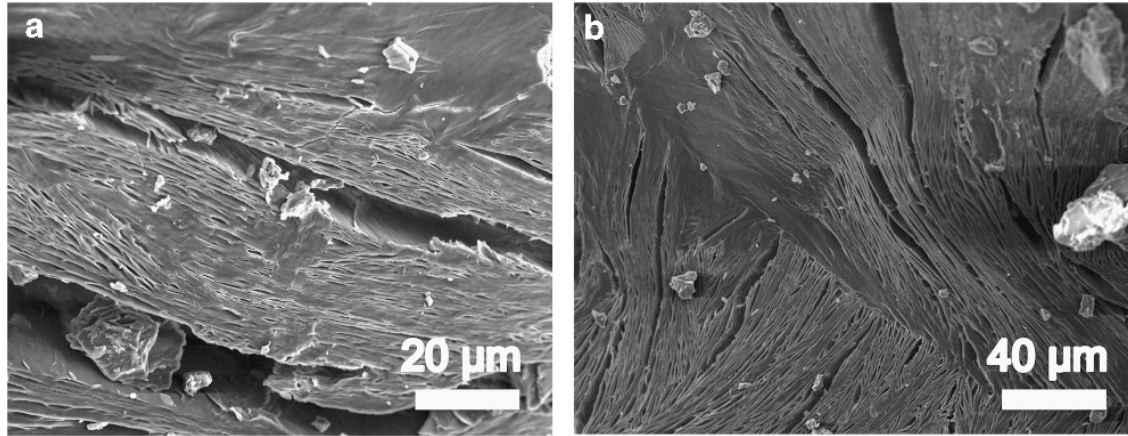

Figure 8 SEM micrographs of the freeze-fractured surface of the 100\% PEG as-cast sample. 

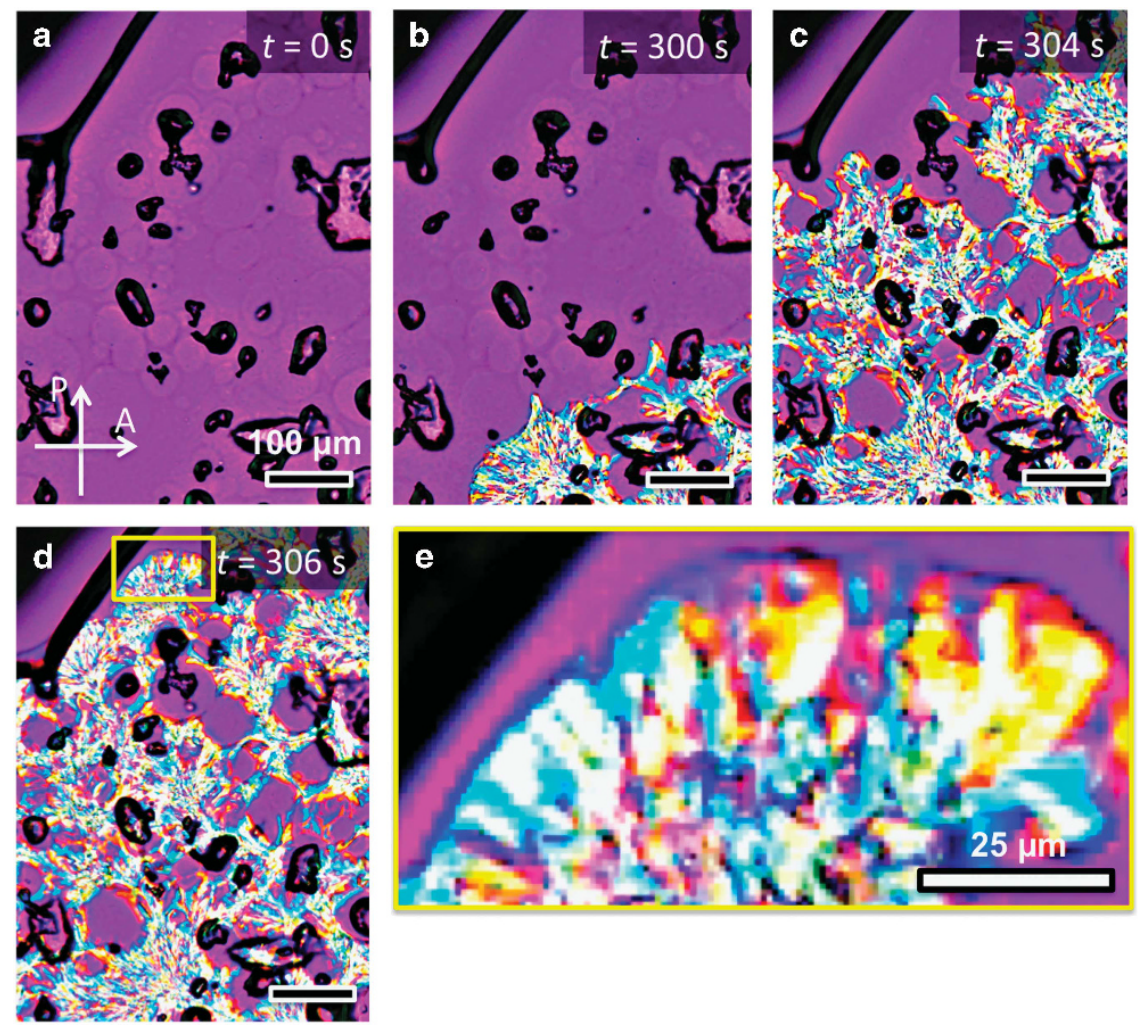

Figure 10 Polarized optical micrographs (a-d) showing the development of crystallization in the DL50 sample melt. The magnified image (e) is cropped from the yellow rectangle in (d) to highlight a feature of a negative spherulite.

acceptable because the amorphous regions of PEG and PDLLA are mixed, namely, no phase-separation should take place due to the miscibility of PEG and PDLLA. However, phase separation is possible during solution casting due to crystallization-induced phase separation. Namely, although two polymers (PEG and PDLLA) are mixed molecularly in the casting solution, the crystallization of PEG causes expulsion of the PDLLA moiety to form the phase-separated domains. Tanaka and $\mathrm{Nishi}^{22}$ have reported on and presented a clear picture of similar round-shaped phase-separation domains that are expelled out from a spherulite during its growth. Such phase separation is not thermodynamically favorable, and the phase-separated domains should be melted away. However, homogenization of the phases would take time. For the particular case of solution casting, if vitrification of the PDLLA-rich phase occurs before homogenization of the phases, crystallization-induced phase separation domains would be frozen in the as-cast sample. On heating of the as-cast DL50 sample, the PDLLArich domains (formed due to the crystallization-induced phase separation, rather than due to thermodynamic phase separation, as stated so above) can be identified, as is in Figure 10a. Again, the PDLLA-rich domains should be homogenized due to the miscibility of PEG and PDLLA, but homogenization may take some time. In the short preheating time $(\sim 2 \mathrm{~min})$ at $70^{\circ} \mathrm{C}$ (above $\left.T_{\mathrm{m}}\right)$ before the course of crystallization in Figure 10a, the PDLLA-rich domains are considered to remain in the sample. These regions remain further during crystallization as shown in Figures $10 \mathrm{a}-\mathrm{d}$ and serve as obstacles to the formation of large PEG spherulites. Thus, crystallization-induced phase separation prevented the formation of large spherulites during melt crystallization, as shown in Figure 10. For the DL20 sample, the amount of PDLLA is low enough that even if crystallization-induced phase separation took place, it would not hinder the formation of large spherulites.

It is also important to note the shape of the voids in the DL50 sample. Unlike the slit-shaped cracks that formed in the 100\% PEG and DL20 samples, many round-shaped voids formed in the DL50 sample, as shown in Figure 7. As stated above, the slit-shaped cracks formed between the crystalline lamellar bundles, and they were likely formed because of volume shrinkage that occurred with secondary crystallization (that is, the development of higher-order crystalline structures in the spherulite interiors). The reason for the formation of roundshaped voids in the DL50 sample may be explained as follows. Many tiny spherulites were formed during solvent evaporation that completely filled the space. Similarly to the final point of crystallization of the $100 \%$ PEG sample (point (b) in the plot of Figure 3), we experimentally found that the polymer concentration of the DL50 casting solution was $\sim 82 \mathrm{wt} \%$ at the time at which the space was completely filled by crystalline regions. This result suggests that the solvent remained in the amorphous region, and those voids were formed by volume contraction on further evaporation of the remaining solvent. If slit-shaped cracks would be formed in the DL50 sample, they were too small to be observed via X-ray CT because such slit-shaped cracks exist in small spherulites, as shown in Figure 10 to be of $\sim 150 \mu$ m diameter.

Existence of cracks in spherulites is not a new finding. In 1961, Inoue reported on fine cracks perpendicular to the boundary of spherulites of polypropylene (PP). 9 'Band cracks' have been found in spherulites of poly(lactic acid) (PLA). ${ }^{23}$ 'Band cracks' are co-centric circular cracks with a periodic interval from the spherulite center. In both cases, the cracks were considered to be formed by volume shrinkage on crystallization. Generally, the formation of polymer spherulites includes several steps: (i) formation of crystalline nucleus; 
(ii) formation of an axialite; (iii) branching and spherical growth of lamellae; (iv) termination of growth by collision of growth fronts of neighbouring spherulites; and (v) ripening of spherulites (that is, volume filling of lamellae in the interior of the spherulites). Steps (i) and (ii) are microscopic phenomena that are out of the scope of the current study. At step (iii), the system consists of tiny solid spherulites sporadically dispersed throughout the polymer melt. At step (iv), collision of growth fronts may result in solid networks of crystalline lamellae that immobilize the system. This type of network is called a 'skeleton of spherulites'. For isotactic polystyrene, Bassett and Olley ${ }^{24}$ successfully observed the 'skeleton' by chemically etching lowmolecular weight components. Volume filling of the skeletal network takes place at step (v). It should be noted that the 'skeleton' cannot shrink so that overall spherulite shrinkage due to the decrease in the volume on further crystallization is prevented. Thus, polymers that exist in-between the 'skeletal network' have to accommodate themselves, giving rise to the cavitation and the slit-like cracks. It is not surprising that cracks in spherulites were first observed with $\mathrm{PP}^{9}$ as it has the large thermal expansivity.

In our systems of both 100\% PEG and its blend with PDLLA, extraordinarily large cracks were found that may be ascribed to additional effects of solvent evaporation that assisted cavity formation during crystallization. As observed in Figure 3, the formation of the 'skeleton' was considered to be accomplished at $900 \mathrm{~min}$, at which point evaporation drastically slows down.

\section{Spherulite morphology}

Figure 5 shows typical cross-sectional views of spherulites, which are highlighted by the dotted curves labeled 'A' and 'B'. Region A shows spoke-like cracks diverging from the middle of a spherulite, and region B contains a 'rose-petal assembly' pattern of cracks. Keller and Waring ${ }^{25}$ previously reported on spoke-like cracks. Later, Khoury and Passaglia $^{14}$ also reported on these two types of patterns by conducting surface replica TEM observation, in which it was noted that these dissimilar patterns originated from the same spherulite. To prove their hypothesis, we digitally sliced one spherulite along different crosssectional directions, as shown in Figure 11. Figure 11a shows a typical spoke-like crack pattern, similar to that observed in region $\mathrm{A}$ in Figure 5. This type of pattern is usually obtained when the crosssectional plane contains the center of the spherulite. The dissimilar patterns are obtained when the spherulite is sliced in off-centered planes. Typical examples are shown in Figures $11 \mathrm{~b}$ and c, which are the cross-sectional views of a single spherulite cut along the broken lines indicated in Figure 11a. Figure 11b shows a typical 'rose-petal assembly' pattern of the cracks, which is similar to a pattern of crystalline lamellae bundles first proposed by Khoury and Passaglia, ${ }^{14}$ and Figure 11c shows a pattern of hyperbolic curves.

These patterns are comprehensively illustrated in Figure 12. As observed in Figures 5 and 11a, the 'spoke-like pattern' seems to be an axialite; however, as this pattern made of cracks rather than crystalline lamellae, it is too large to be the axialite. As stated above, the crack is an indication of the lamellar bundle orientation so that the patterns observed in Figure 11 should be realized in 3D space, in which the spherical assembly of lamellar bundles is accommodated. In fact, X-ray CT 3D images confirmed that the cracks were curved. To form a spherical aggregate of lamellar bundles, the cracks should branch out and curl. Thus, the axialite is formed. Figure $12 \mathrm{~d}$ has cylindrical symmetry around the dotted line, which is the rotation axis. One can
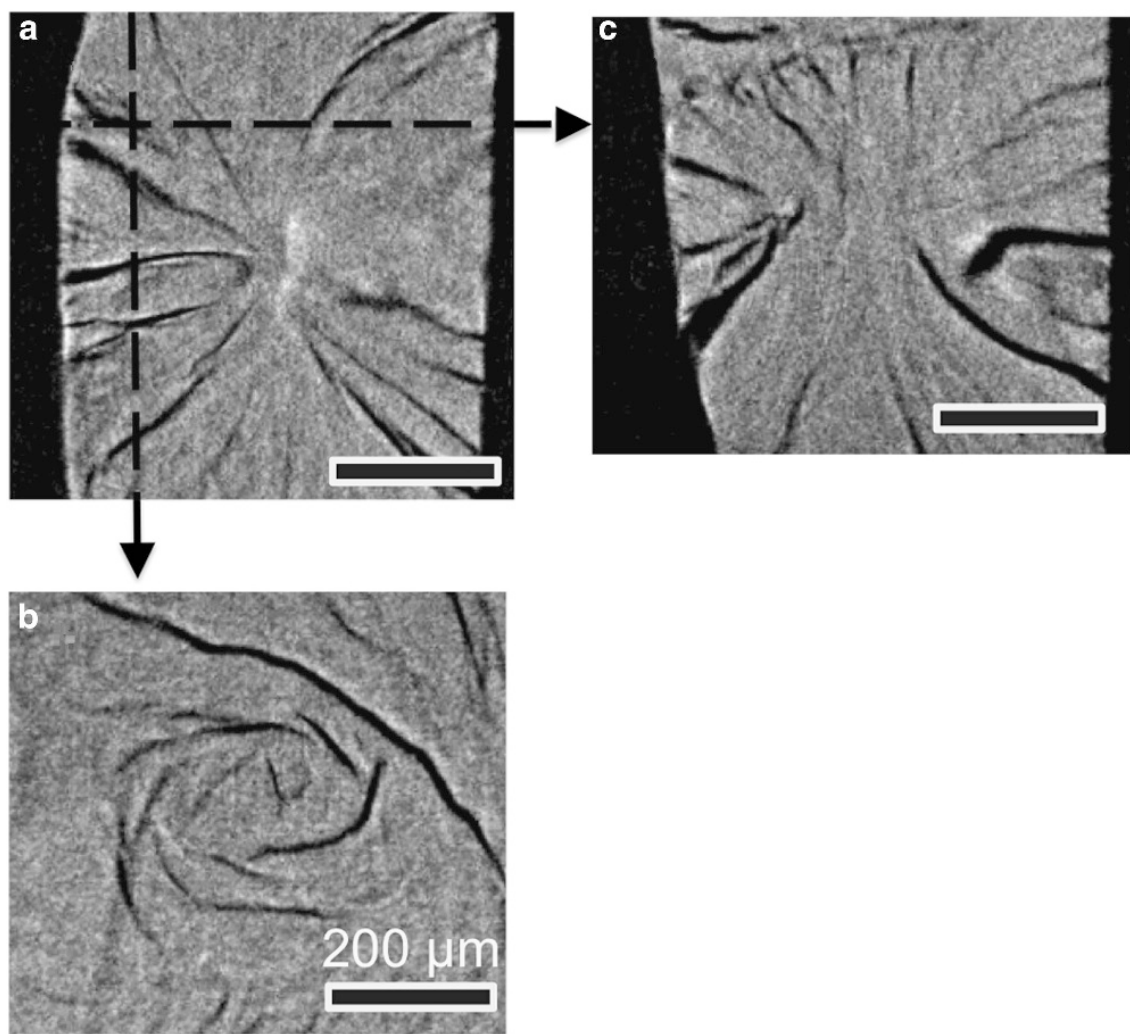

Figure 11 Typical cross-sectional images of a single spherulite obtained by conducting digital slicing of the 3D data obtained using X-ray CT. (a) A crosssection featuring a central portion of the spherulite. (b, c) Cross-sectional views of planes that are perpendicular to the image shown in (a), as indicated by the broken lines. The broken lines show different cutting plane positions: vertical to provide the view (b) and horizontal to provide the view (c). 


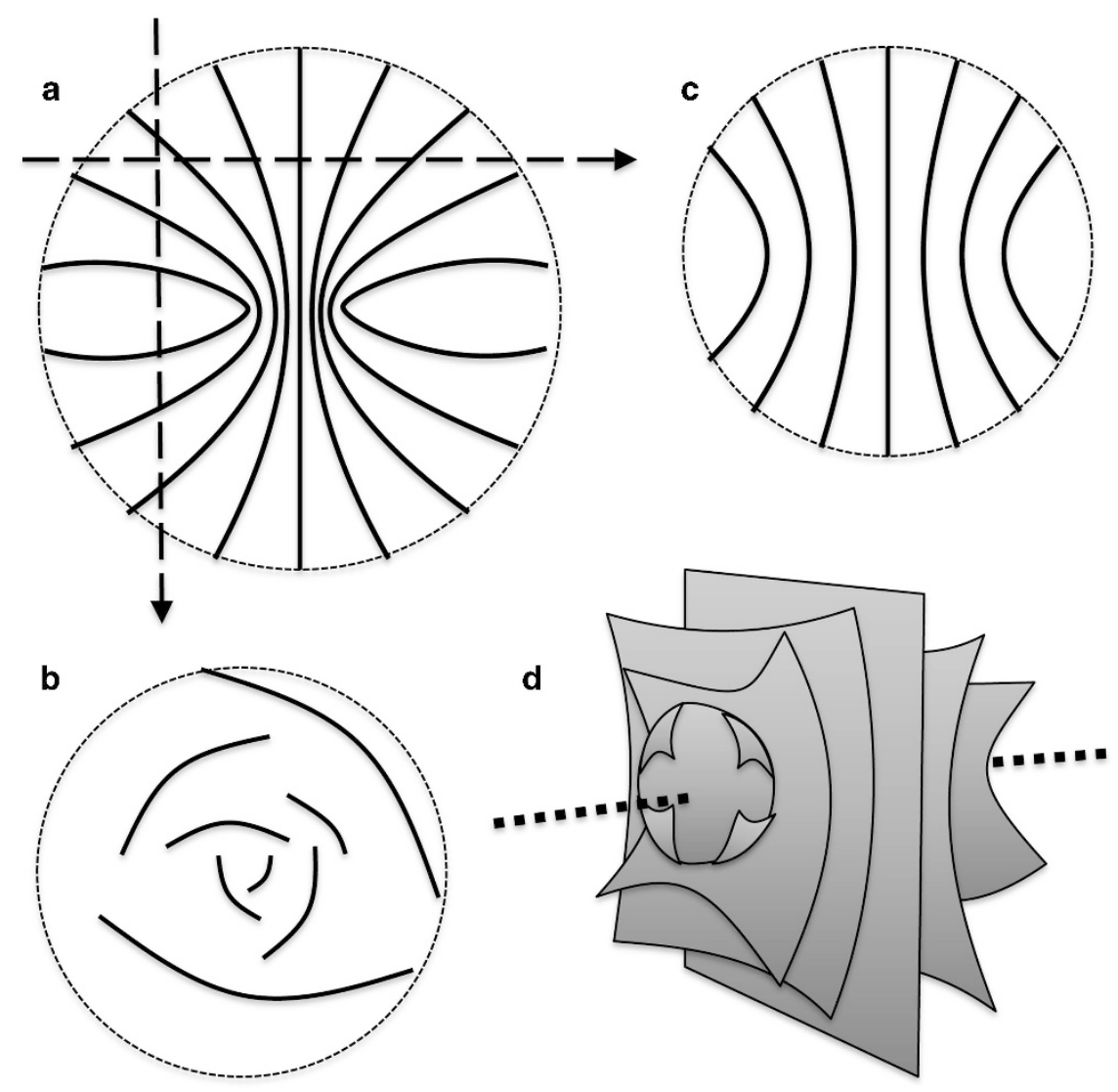

Figure 12 Schematic representations of the cross-sectional views $(\mathbf{a}-\mathbf{c})$ that correspond to the experimentally-observed cross-sections shown in Figure 11. Illustration (d) represents the 3D model of crack assembly in a spherulite. Here, each crack (black solid line) in illustrations (a-c) represents a curved sheet. The broken lines show different cutting plane positions: vertical to provide the view (b) and horizontal to provide the view (c).

see that the cross-section that includes the rotation axis becomes a 'spoke-like pattern,' as shown in Figure 12a. By contrast, the crosssection perpendicular to the rotation axis must have rotational symmetry, but these cracks cannot be circles as they only slightly curl. Therefore, the cross-section is a 'rose-petal assembly' pattern, as shown in Figure 12b. The model in Figure 12d also explains the pattern of hyperbolic curves in Figure 11c, which is ascribed to the cross-section parallel to the rotation axis. If the distance of the crosssection from the rotation axis is increased, the number of cracks becomes smaller. Consequently, it is concluded that the interpretation illustrated in Figure 12d agrees with the experimental observation shown in Figure 11. In the X-ray CT observations, the 'axialitic' pattern observed in Figures 5 and 11a is meaningful and interesting, although it is much larger than the actual axialite that exists in the central part of the spherulite.

As the rotation axis passes through the center of the spherulite, the center of the 'spoke-like pattern' should be coincident with the spherulite center. In Figure 11a, one can see a bright (white) region in the central part of the 'spoke-like pattern'. In general, the pixel intensity in X-ray CT images is proportional to the X-ray absorption coefficient. ${ }^{16}$ The bright center in Figure 11a suggests the existence of a region with high electron density in the spherulite center. A similar bright region was found at the central part of each spherulite examined in the current study. The bright region can be recognized as a small 'ribbon', which is a thin bundle of lamellar crystals. In the case of polymer spherulites, two possibilities other than the axialite could be the origin of the bright region. One is a foreign particle

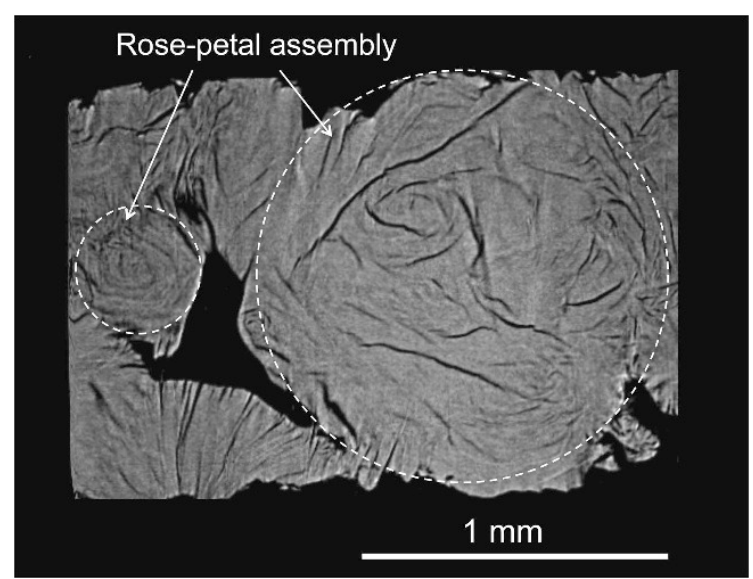

Figure $13 \mathrm{X}$-ray CT image highlighting the substrate-side surface of the $100 \%$ PEG as-cast sample.

nucleus. If the foreign particle is dust, it is usually round-shaped (such as a piece of silicate soil) and therefore does not appear as a 'ribbon'. Thus, this possibility is not the case. Another possibility for the object at the center of a spherulite is a crystalline nucleus consisting of high-molecular weight polymer chains with high crystallinity and high electron density. However, this alternative is less plausible as a single nucleus is considered to be much smaller than that can be observed 
with the spatial resolution of $\sim 3 \mu \mathrm{m}$ of X-ray CT. Therefore, it is reasonable to consider that the bright regions in these images contain highly bundled lamellae with high electron density. Therefore, the 'ribbon' shape of the bright region found in the center of the spherulite (Figure 11a) can be ascribed to the axialite.

In our observation, the bright region at the spherulite center is typically found in the interior of the polymer film. As observed in the inset of Figure 5, the film surface gradually undulates, reflecting the round shape of the spherulite. This topography indicates that the growth fronts of the spherulites were terminated at the surface of the as-cast sample. Figure 13 highlights the substrate-side surface of the 100\% PEG as-cast sample. Two broken circular curves with $\sim 1.2 \mathrm{~mm}$ and $0.5 \mathrm{~mm}$ diameter highlight a typical 'rose-petal assembly' pattern, which corresponds to the spherulite intersection perpendicular to the rotation axis (as illustrated in Figure 12b). Therefore, the region is considered to be distant from the spherulite center, which indicates that nucleation did not take place at the substrate-side surface. To summarize, nucleation primarily takes place spontaneously in the interior of the casting solution and is not triggered by the substrate surface.

\section{CONCLUSIONS}

Unusually large spherulites were formed in the as-cast samples of 100\% PEG and DL20 blend from dichloromethane solutions. Moreover, large cracks were successfully found in the PEG spherulites using $\mathrm{X}$-ray CT. It was found that the cracks run along constituent bundles of the lamellar crystal and can therefore serve as indicators of the lamellar orientation. X-ray CT revealed details of the spherulites in three dimensions. PEG spherulites were well modeled as a curled bundle of lamellar crystallites, as shown in Figure 12d. In addition, the centers of the PEG spherulites were located in the interior of the film rather than on the substrate-side or free surfaces.

\section{ACKNOWLEDGEMENTS}

This study was partially supported by the Grant-in-Aid for Scientific Research on Innovative Areas, 'New Polymeric Materials Based on Element-Blocks' (No. 25102524), from the Ministry of Education, Culture, Sports, Science, and Technology of Japan.

1 Coish, R. A., Taylor, L. A. The effects of cooling rate on texture and pyroxene chemistry in DSDP Leg 34 basalt: A microprobe study. Earth Planet. Sci. Lett. 42, 389-398 (1979).

2 Davis, B. K., McPhie, J. Spherulites, quench fractures and relict perlite in a Late Devonian rhyolite dyke, Queensland, Australia. J. Volcanol. Geothermal Res. 71 $1-11$ (1996).
3 Fowler, A. D., Berger, B., Shore, M., Jones, M. I., Ropchan, J. Supercooled rocks: development and significance of varioles, spherulites, dendrites and spinifex in Archaean volcanic rocks, Abitibi Greenstone belt, Canada. Precambrian Res. 115 311-328 (2002)

4 Prasad, P. B. V. Crystal growth of fatty acids from melt: $\mathrm{CH} 3(\mathrm{CH} 2) 14 \mathrm{COOH}$ and $\mathrm{CH} 3$ (CH2)16COOH. Crystal Res. Technol. 19, 1549-1552 (1984).

5 Price, F. P., Fritzsche, A. K. Kinetics of spherulite growth in cholesteryl esters. J. Phys. Chem. 77, 396-399 (1973)

6 Khoury, F. The Spherulitic crystallization of isotactic polypropylene from solution: on the evolution of monoclinic spherulites from dendritic chain-folded crystal precusors. $J$. Res. Natl Bureau Standard - A. Phys. Chem. 70A, 29 (1966).

7 Wang, Z., Alfonso, G. C., Hu, Z., Zhang, J., He, T. Rhythmic growth-induced ringbanded spherulites with radial periodic variation of thicknesses grown from poly $(\varepsilon-$ caprolactone) solution with constant concentration. Macromolecules 41, 7584-7595 (2008).

8 Gránásy, L., Pusztai, T., Warren, J. A., Douglas, J. F., Börzsönyi, T., Ferreiro, V. Growth of 'dizzy dendrites' in a random field of foreign particles. Nat. Mater. 2 92-96 (2003).

9 Inoue, M. Spherulitic crystallization and cracking during heat aging of polypropylene J. Polymer Sci. 55, 443-450 (1961).

10 Sand, K. K., Rodriguez-Blanco, J. D., Makovicky, E., Benning, L. G., Stipp, S. L. S. Crystallization of $\mathrm{CaCO} 3$ in water-alcohol mixtures: spherulitic growth, polymorph stabilization, and morphology change. Cryst. Growth Des. 12, 842-853 (2011).

11 Schultz, J. M. Polymer Crystallization: The Development of Crystalline Order in Thermoplastic Polymers (American Chemical Society, Washington DC, USA 2001).

12 Woodward, A. E. Atlas of Polymer Morphology (Hanser Publishers, Munich, Vienna, New York, 1989).

13 Bassett, D. C., Vaughan, A. S. On the lamellar morphology of melt-crystallized isotactic polystyrene. Polymer 26, 717-725 (1985)

14 Khoury, F., Passaglia, E. in Treatise on Solid State Chemistry (ed. Hannay N. B.) Ch. 6, 335-496 (Springer, New York, USA, 1976).

15 Sperling, L. H. Introduction to Physical Polymer Science (John Wiley \& Sons, New York USA, 1986).

16 Nishikawa, Y., Baba, S., Takahashi, M. Optimization of X-Ray computerized tomography for polymer materials. Int. J. Polymeric Mater. Polymeric Biomater. 62 295-300 (2012).

17 Nishikawa, Y., Hatanaka, Y., lizuka, S., Takahashi, M. Survey of contrasts of polymers under a high-contrast X-ray computerized tomography. Polymer 53, 4287-4292 (2012)

18 Tien, N.-D., Hoa, T.-P., Mochizuki, M., Saijo, K., Hasegawa, H., Sasaki, S., Sakurai, S Higher-order crystalline structures of poly(oxyethylene) in poly(d,l-lactide)/poly (oxyethylene) blends. Polymer 54, 4653-4659 (2013).

19 Tien, N.-D., Sasaki, S., Masunaga, H., Shimizu, N., Igarashi, N., Sakurai, S. Smallangle X-ray scattering studies on melting and recrystallization behaviors of poly (oxyethylene) crystallites in poly(d,I-lactide)/poly(oxyethylene) blends. Polymer $\mathbf{5 5}$ 2562-2569 (2014)

20 Herman, G. T. Fundamentals of Computerized Tomography: Image Reconstruction from Projections. Advances in Pattern Recognition, 2nd edn (Springer, London, UK, 2009).

21 Brandrup, J., Immergut, E. H. Polymer Handbook, 3rd edn (Wiley-Interscience, Hoboken, NJ, USA, 1989).

22 Tanaka, H., Nishi, T. New Types of Phase Separation Behavior during the Crystallization Process in Polymer Blends with Phase Diagram. Phys. Rev. Lett. 55, 1102-1105 (1985).

23 Fraschini, C., Plesu, R., Sarasua, J.-R., Prud'homme, R. E. Cracking in polylactide spherulites. J. Polymer Sci. Part B 43, 3308-3315 (2005).

24 Bassett, D. C., Olley, R. H. On the lamellar morphology of isotactic polypropylene spherulites. Polymer 25, 935-943 (1984).

25 Keller, A., Waring, J. R. S. The spherulitic structure of crystalline polymers. Part III. Geometrical factors in spherulitic growth and the fine-structure. J. Polymer Sci. 17 447-472 (1955) 